\title{
DISKS IN $E^{3}$. I. SUBSETS OF DISKS HAVING NEIGHBORHOODS LYING ON 2-SPHERES \\ BY
}

\author{
RALPH J. BEAN(1)
}

1. Introduction. It is well known that every simple closed curve in $E^{2}$ may be approximated arbitrarily closely from either side by a polyhedral simple closed curve and that every two simple closed curves in $E^{2}$ are equivalently imbedded, i.e., there exists a homeomorphism of $E^{2}$ onto itself taking one onto the other, [14]. The analogous statements for 2-spheres in $E^{3}$ are false. There are examples of 2-spheres in $E^{3}$ which cannot be homeomorphically approximated from the side (see $[1 ; 5 ; 6 ; 10]$ ) and examples of 2 -spheres in $E^{3}$ whose imbeddings are not equivalent to the imbedding of a polyhedral 2 -sphere (see $[1 ; 5 ; 10]$ ).

It has been proven by Bing [3] that a 2 -sphere in $E^{3}$ may be approximated arbitrarily closely by a polyhedral 2 -sphere and even that the 2-sphere may be "almost" approximated from the side [2], i.e., that the approximating 2-sphere may be made to lie, except for a finite number of small subdisks, on either side of the original 2-sphere. These approximation theorems have led to what may be called a "break through" in the study of surfaces in $E^{3}$.

Now the study of disks in $E^{3}$ would be greatly aided if we knew that each disk in $E^{3}$ lies on some 2-sphere in $E^{3}$, for then Bing's theorem on "almost" approximating a 2-sphere from the side would apply to disks. It is well known that each arc in $E^{2}$ lies on a simple closed curve in $E^{2}$; however, Bing has given an example of a disk in $E^{3}$ which lies on no 2-sphere [7]. This example is discussed in $\$ 3$ and pictured in Figure 1. Joseph Martin has given necessary and sufficient conditions for a disk in $E^{3}$ to lie on a 2 -sphere in $E^{3}$ [11].

In Part I of this paper we will develop necessary and sufficient conditions for certain subsets of disks to have neighborhoods on the disk which lie on 2-spheres. This work was motivated by a question asked by Bing in [9] which is answered in $\S 3$.

We will also prove that if a disk has only one wild point, which is in the interior of the disk, then the disk lies on a 2-sphere in $E^{3}$. An example is given to show that with two wild points, the disk need not lie on a 2 -sphere.

Presented to the Society, January 22, 1962, under the title On subsets of a disk which contain all the wild points of the disk; received by the editors December 15, 1962 and, in revised form, March 20, 1963.

(1) This work was done while the author held a National Science Foundation Cooperative Graduate Fellowship. The author expresses his graditude to Guido Lehner, who assisted him greatly in the preparation of this manuscript. 


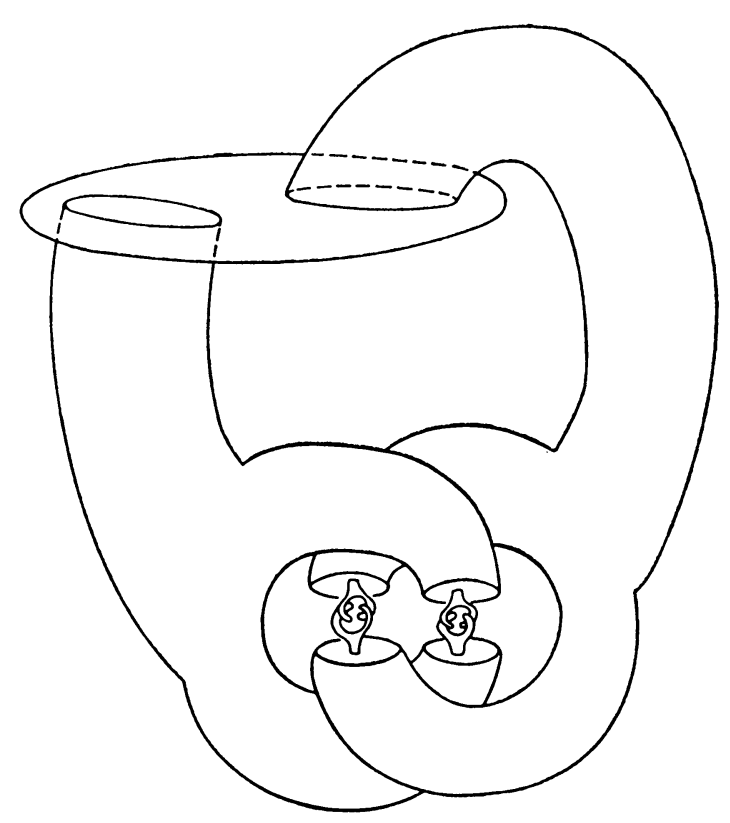

Figure 1

In Part II we will prove that if $D$ is a disk in $E^{3}$ such that all the wild points of $D$ are in Int $D$, then $D$ "almost" lies on a 2 -sphere in $E^{3}$, i.e., given $\varepsilon>0$, there exists a finite number of mutually exclusive subdisks $\left\{D_{i}\right\}_{i=1}^{N}$ of Int $D$ with $\operatorname{diam} D_{i}<\varepsilon$ and such that $D-\sum_{i=1}^{N}\left(\operatorname{Int} D_{i}\right)$ lies on a 2 -sphere in $E^{3}$. This result will then be extended to more general disks and some consequences of the theorem will be indicated.

2. Definitions and notation. By $n$-ball we mean the set

$$
\left\{\left(x_{1}, x_{2}, \cdots, x_{n}\right) \mid x_{1}^{2}+x_{2}^{2}+\cdots+x_{n}^{2} \leqq 1\right\}
$$

in $E^{n}$. An $n$-cell $C^{n}$ is any set homeomorphic to the $n$-ball and an $(n-1)$-sphere $S^{n-1}$ is any set homeomorphic to the boundary of the $n$-ball. Note that an $n$-sphere is homeomorphic to the one-point compactification of $E^{n}$. The interior of an $n$-cell Int $C^{n}$ is the set of those points which, under any homeomorphism from the $n$-cell to the $n$-ball, have their images in the point-set interior of the $n$-ball in $E^{n}$. Note that this may not coincide with the point-set interior of the $n$-cell, for the point-set interior depends on the space in which the $n$-cell is imbedded, while the interior is invariant. Likewise, the boundary of an $n$-cell $\mathrm{Bd}^{n}$ is the set $C^{n}-$ Int $C^{n}$, and may not coincide with the point-set boundary.

An $n$-annulus is a set homeomorphic to the Cartesian product of an $(n-1)$ sphere $S^{n-1}$ and the unit interval $[0,1]$. If an $(n-1)$-sphere $S^{n-1}$ is imbedded in $E^{n}$ (or in the interior of an $n$-cell) we will use Int $S^{n-1}$ to denote the bounded 
complementary domain of $S^{n-1}$ (or the complementary domain of $S^{n-1}$ which does not contain the boundary of the $n$-cell) and Ext $S^{n-1}$ to denote the other complementary domain. If $S^{\prime}$ and $S$ are two $(n-1)$-spheres in $E^{n}$ (or in an $n$-cell) with $S^{\prime} \subset \operatorname{Int} S,\left[S^{\prime}, S\right]$ will denote (Ext $\left.S^{\prime} \cap \operatorname{Int} S\right) \cup S^{\prime} \cup S$.

An arc is 1-cell, a disk is a 2-cell, and a simple closed curve is a 1-sphere.

A geometric complex is a metric space which is the sum of a locally finite collection of simplices such that if two simplices intersect, the intersection is a face of each. A set $P$ is called a polyhedron in a geometric complex $C$ if it is equal to a subcomplex of some subdivision of $C$. A point set $K$ in $C$ is locally polyhedral at a point $p$ of $K$ if there is an open set $U$ containing $p$ such that $\bar{U} \cap K$ is a polyhedron in $C$.

We will say that a subset $K$ of $E^{n}$ is tamely imbedded (or tame) if there is a homeomorphism of $E^{n}$ onto itself which takes $K$ onto a polyhedron. $K$ will be called locally tamely imbedded if for each point $p$ of $K$, there is a neighborhood $N$ of $p$ and a homeomorphism $h_{p}$ of $\bar{N}$ onto a polyhedron in $E^{n}$ such that $h_{p}(\bar{N} \cap K)$ is a polyhedron. Any point of $K$ where $K$ fails to be locally tamely imbedded will be called a wild point of $K$. Bing in [8] and, independently, Moise in [12] have proven that in a 3-manifold, every locally tamely imbedded closed set $K$ is tamely imbedded.

A subset $X$ of an $n$-dimensional metric space $M$ will be called cellular if there exists a sequence of $n$-cells $\left\{C^{n}\right\}$ in $M$ such that $C_{i+1} \subset \operatorname{Int} C_{i}$ and $X=\bigcap_{i=1}^{\infty} C_{i}$. It is well known that these $n$-cells may be taken to be bi-collared and such that [Bd $\left.C_{i+1}, \mathrm{Bd} C_{i}\right]$ is an $n$-annulus for each $i$ [9].

A set of $n$ distinct points in $E^{3}(n>3)$ will be said to be in general position if and only if no four of the points lie on a plane in $E^{3}$. We will say that the polyhedron $K$ is in general position with respect to the polyhedron $K^{\prime}$ if and only if no vertex of $K$ is a vertex of $K^{\prime}$ and the sum of the vertices of $K$ and the vertices of $K^{\prime}$ is in general position. It is well known that any finite system of points in $E^{3}$ can be brought into general position by an arbitrarily small displacement (see, for example, Theorem 2 of [13]). It is evident that if $K$ is a polyhedron whose vertices are in general position and $K^{\prime}$ is an arbitrary polyhedron, then $K^{\prime}$ may be brought into general position with respect to $K$ by an arbitrarily small displacement of the vertices of $K^{\prime}$, i.e., an arbitrarily small displacement (piecewise linear) of $K^{\prime}$.

3. Cellular subsets which have neighborhoods lying on 2-spheres. We are now in a position to prove Theorem 1. From this theorem and an example first described by Bing we immediately get an answer to the following question. This question was asked by Bing at the National Science Foundation Institute for Graduate Students in Topology, Summer, 1961 [9].

Question (BING). Suppose $D$ is a disk in $E^{3}$ and $\alpha$ is an arc in Int $D$. Is there a subdisk $D^{\prime}$ of $D$ such that $\alpha \subset \operatorname{Int} D^{\prime}$ and $D^{\prime}$ lies on a 2 -sphere in $E^{3}$ ? 
THeOREM 1. Let $D$ be a disk in $E^{3}$ and let $X$ be a cellular subset of $D$, $X \subset \operatorname{Int} D$, such that $X$ contains all the wild points of $D$. Let $U$ be a neighborhood of $X$ in $D$. Then there exists a subdisk $D^{\prime}$ of $D$ in $U$ and a homeomorphism $h$ of $E^{3}$ onto itself such that $h\left(D^{\prime}\right)=D$ and $h$ is the identity on some open set $V$ which contains $X$.

Proof. Since $X$ is cellular in $D$, there exist disks, $D_{1}$ and $D_{2}$, such that

$$
X \subset \operatorname{Int} D_{2} \subset D_{2}^{\prime} \subset \operatorname{Int} D_{1} \subset D_{1} \subset U
$$

and such that $\left[\mathrm{Bd} D_{i}, \mathrm{Bd} D\right]$ is a 2-annulus $(i=1,2)$. We define a continuous, non-negative function $f$ on $D$ such that $f(x)=0$ for $x \in \operatorname{Ext} D_{2}$ and $f(x)>0$ for $x \in \operatorname{Int} D_{2}$. This function might be defined as follows: $f(x)=\delta\left(x\left[\operatorname{Bd} D_{2}, \operatorname{Bd} D\right]\right)$, where $\delta$ is the distance function in $E^{3}$. Then, using Bing's Approximation Theorem for Surfaces (Theorem 8 of [3]), we can get a new disk $E$ such that $E \cap D \supset\left[\mathrm{Bd} D_{2}, \mathrm{Bd} D\right]$ and $E$ is locally tame at each point. From [8] and [12] we know that $E$ is tamely imbedded.

We know that there exists a homeomorphism $g$ of $E^{3}$ onto itself which takes $E$ onto the disk $\left\{\left(x_{1}, x_{2}, 0\right) \mid x_{1}^{2}+x_{2}^{2} \leqq 1\right\}, \operatorname{Bd} D_{2}$ onto $\left\{\left(x_{1}, x_{2}, 0\right) \mid x_{1}^{2}+x_{2}^{2}=1 / 2\right\}$, and $\operatorname{Bd} D_{1}$ onto $\left\{\left(x_{1}, x_{2}, 0\right) \mid x_{1}^{2}+x_{2}^{2}=3 / 4\right\}$.

We will now describe a space homeomorphism $f$ which takes $g\left(D_{1}\right)$ onto $g(D)$ and is fixed on some $\varepsilon$-neighborhood of $g\left(D_{2}\right)$. If we define $V$ by the requirement that $g(V)$ is this $\varepsilon$-neighborhood, then, clearly, $h=g^{-1} f g$ will be the homeomorphism required by the theorem.

Consider the annulus $\left[g\left(\operatorname{Bd} D_{1}\right), g(\operatorname{Bd} D)\right]=A$ and define $\varepsilon>0$ by the requirement that $\delta\left(A, g\left(D_{2}\right)\right)>2 \varepsilon$. The description $f$ will be given using cylindrical coordinates $(r, \omega, z)$ for $E^{3}$. First, in the $r \omega$-plane, for each fixed $\omega\left(\omega=\omega_{\alpha}\right)$, let $f$ take the intervals $\left\{(r, \omega) \mid 3 / 4-\varepsilon \leqq r \leqq 3 / 4, \omega=\omega_{\alpha}\right\}$ and $\left\{(r, \omega) \mid 3 / 4 \leqq r \leqq 1+\varepsilon, \omega=\omega_{\alpha}\right\}$ linearly onto the intervals $\{(r, \omega) \mid 3 / 4-\varepsilon \leqq r \leqq 1$, $\left.\omega=\omega_{\alpha}\right\}$ and $\{(r, \omega) \mid 1 \leqq r \leqq 1+\varepsilon\}$ respectively and let $f$ be the identity for $r \leqq 3 / 4-\varepsilon$ and $r \geqq 1+\varepsilon$. Denote the image of $(r, \omega, z)$ by $\left(r^{\prime}, \omega, z\right)$. Next, for each fixed $\omega\left(\omega=\omega_{\alpha}\right)$, and for $3 / 4-\varepsilon \leqq r \leqq 1+\varepsilon$, let $f$ take the intervals from $\left(r, \omega_{\alpha}, 0\right)$ to $\left(7 / 8, \omega_{\alpha}, \varepsilon\right)$ and from $\left(r, \omega_{\alpha}, 0\right)$ to $\left(7 / 8, \omega_{\alpha},-\varepsilon\right)$ linearly onto the intervals from $\left(r^{\prime}, \omega_{\alpha}, 0\right)$ to $\left(7 / 8, \omega_{\alpha}, \varepsilon\right)$ and from $\left(r^{\prime}, \omega_{\alpha}, 0\right)$ to $\left(7 / 8, \omega_{\alpha},-\varepsilon\right)$ respectively. Last, at all other points of $E^{3}$, let $f$ be the identity. It is an elementary matter to show that $f$ is a space homeomorphism with the required properties and our proof is complete.

Corollary 1. If $D$ is a disk in $E^{3}$ and $X$ is a cellular subset of $D, X \subset \operatorname{Int} D$, such that $X$ contains all the wild points of $D$, then there exists a neighborhood of $X$ in $D$ which lies on a 2-sphere if and only if D lies on a 2-sphere.

Proof. This is a direct application of Theorem 1 . 
EXAmple (Bing). In [7], Bing gave an example of a disk in $E^{3}$ with the following properties. (A) There exists an arc in the interior of the disk which contains all the wild points of the disk, and (B) the disk lies on no 2-sphere in $E^{3}$. Since the arc is cellular in Int $D$, this disk and arc provide a negative answer to the question above. This example is pictured in Figure 1.

4. Disks lying on no 2-sphere. Note that the disk of the preceding example has uncountably many wild points, so one might naturally ask if this is necessary. This question becomes especially interesting in view of the following result.

Corollary 2. Let $D$ be a disk in $E^{3}$ such that $D$ has only one wild point $p$, $p \in \operatorname{Int} D$. Then $D$ lies on a 2-sphere in $E^{3}$.

Proof. Bing has proven in [4] that if $p$ is an interior point of a disk $D$ in $E^{3}$, then there exists a neighborhood $U$ of $p$ in $D$ which lies on a 2-sphere. The point $p$ is clearly a cellular subset of $D$ so by Corollary $1, D$ lies on a 2 -sphere.

The question asked above is solved by the following.

ExAmple. This example is a modification of an arc given by Fox and Artin in [10] (see Figure 2). It is clear that this arc may be put on a 2-sphere by thicken-
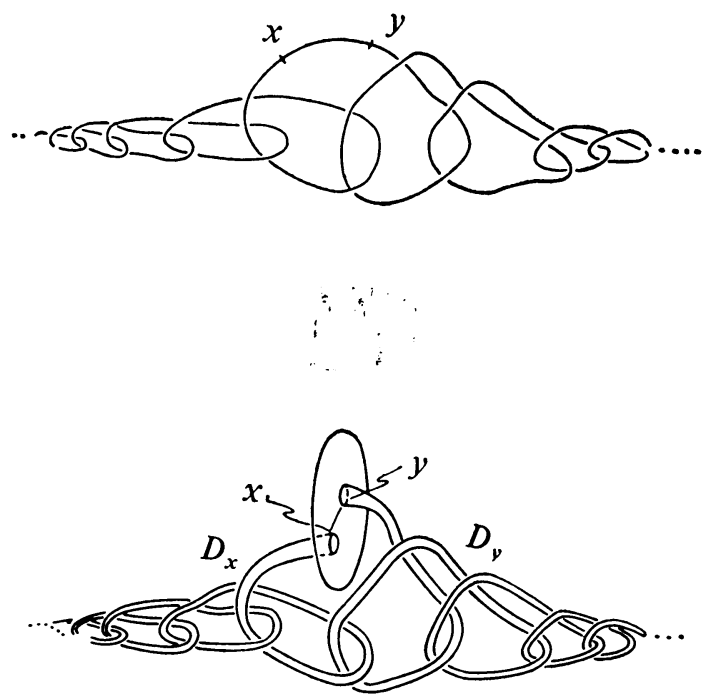

FIGURE 2

ing the arc to get a 2-sphere, but consider this alternate construction. Starting from point $x$, thicken the arc to the left to get a disk $D_{x}$, and from the point $y$, thicken the arc to the right to get a disk $D_{y}$. We then have two disks connected by the arc $\overline{x y}$. If we put this arc on a disk in such a way that the disks $D_{x}$ and $D_{y}$ come out of it from opposite sides we get the construction pictured in Figure 2. This disk has only two wild points, the end points of the original arc, yet $D$ lies 
on no 2-sphere. To see this consider $\operatorname{Bd} D$. $\operatorname{Bd} D$ cannot be shrunk to a point in $\left(E^{3}-D\right) \cup \operatorname{Bd} D[10]$. If $D$ were on a 2 -sphere, $\operatorname{Bd} D$ would bound a disk in $E^{3}-$ Int $D$ and could be shrunk to a point on this disk.

5. Noncellular subsets which have neighborhoods lying on 2-spheres. One might wonder if Corollary 1 of the last section holds for continua $X$ which are not cellular. In this section we will prove that it does, in fact, hold for any continuum. In Theorem 2 we will consider the simplest case: $X$ is a simple closed curve. The techniques developed in the proof of this theorem will permit immediate extension.

THEOREM 2. Let $D$ be a disk in $E^{3}$ and let $X$ be a simple closed curve, $X \subset$ Int $D$, such that $X$ contains all the wild points of $D$. Then there exists $a$ neighborhood $U$ of $X$ in $D$ which lies on a 2-sphere if and only if $D$ lies on a 2-sphere.

Proof. One direction of this theorem is trivial. If $D$ lies on a 2 -sphere, then $U$ may be taken as Int $D$.

To prove that the existence of a neighborhood $U$ of $X$ in $D$ lying on a 2-sphere implies that $D$ lies on a 2 -sphere we note that $X$ bounds a subdisk $D^{\prime}$ of $D$ which is cellular in $D$. In view of this and Corollary 1 we need only prove that if there exists a neighborhood $U$ of $X$ in $D$ lying on a 2 -sphere, then there exists a subdisk $D_{2}$ of $D$ such that $X \subset \operatorname{Int} D_{2}$ and $D_{2}$ lies on a 2-sphere.

Let $D_{1}$ and $D_{2}$ be two subdisks of $D$ such that $\operatorname{Bd} D_{i} \subset U$ for $i=1,2$, $D_{1} \subset \operatorname{Int} D^{\prime} \subset D^{\prime} \subset \operatorname{Int} D_{2}$, and such that $\left[\operatorname{Bd} D_{1}, \operatorname{Bd} D_{2}\right]$ is an annulus in $U$. Note that by Theorem 8 of [8],

$$
\left(D-\left[\operatorname{Bd} D_{1}, \operatorname{Bd} D_{2}\right]\right) \cup \operatorname{Bd} D_{1} \cup \operatorname{Bd} D_{2}
$$

may be assumed to be polyhedral. Also assume that $U$ lies on a 2-sphere $S$, which, by Theorem 7 of [3] may be taken to be locally polyhedral at all points of

$$
\left(S-\left[\operatorname{Bd} D_{1}, \operatorname{Bd} D_{2}\right]\right) \cup \operatorname{Bd} D_{1} \cup \operatorname{Bd} D_{2} .
$$

Bd $D_{2}$ bounds two disks in $S$ and we denote by $E$ that one which does not contain $X$. Finally, we may also assume that $E$ is in general position with respect to $D_{1}$. In order to prove that $D_{2}$ lies on a 2 -sphere we will modify $E$ to get a new disk $F$ such that $D_{2} \cap F=\operatorname{Bd} D_{2}$. The sphere will then be $D_{2} \cup F$.

Since $E \cap \operatorname{Bd} D_{1}=D_{1} \cap \mathrm{Bd} E=\varnothing$ and since $E$ and $D_{1}$ are in general position, $E \cap D_{1}$ is the sum of a finite number of simple closed curves. We will show now how to modify $E$ to get a new disk $E^{\prime}$ such that $E^{\prime} \cap D_{1}$ consists of at least one less simple closed curve and such that Int $E^{\prime} \cap\left[\operatorname{Bd} D_{1}, \operatorname{Bd} D_{2}\right]=\varnothing$. If we repeat this step as many times as necessary, the final disk $E^{(k)}$ will be the disk $F$ we are seeking. (For a visual aid, see Figure 3.) 


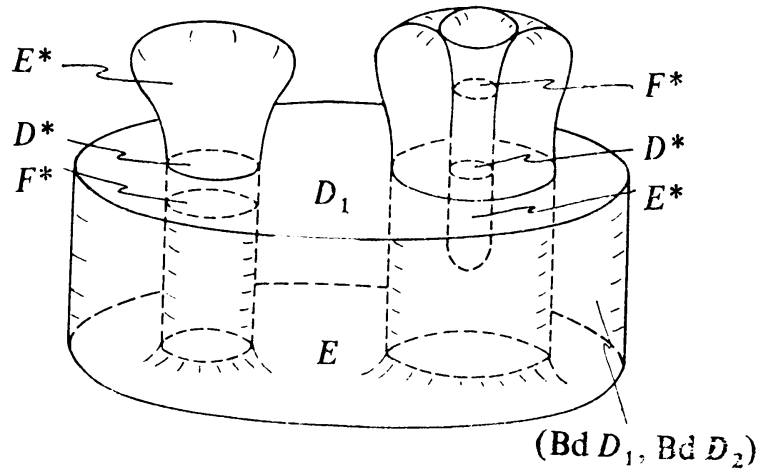

Figure 3

Consider any "interior" simple closed curve $J$ of $E \cap D_{1}$. By this we mean a curve which bounds a subdisk $E^{*}$ of Int $E$ and a subdisk $D^{*}$ of Int $D_{1}$ such that Int $D^{*} \cap E=\varnothing$. It is clear that if $E \cap D_{1} \neq \varnothing$, there will exist at least one "interior" simple closed curve. We will replace $E^{*}$ by the disk $D^{*}$, then push this disk slightly off of $D_{1}$ so that the modified $E$ has at least one less component in its intersection with $D_{1}$. (In Figure $3, E^{*}$ is replaced by $F^{*}$.)

THEOREM 3. Let $X$ be a closed, connected subset of the interior of a disk $D$ in $E^{3}$ such that $X$ contains all the wild points of $D$. Then there exists a neighborhood $U$ of $X$ in $D$ which lies on a 2-sphere if and only if D lies on a 2-sphere.

The proof follows from the fact that every closed connected subset $X$ of the interior of $D$ may be written as the intersection of a sequence of sets $X=\bigcap_{i=1}^{\infty} T_{i}$ where each $T_{i}$ is a disk with a finite number of disjoint open subdisks removed from its interior and $T_{i+1} \subset T_{i}$. Then, since every neighborhood of $X$ in $D$ must be a neighborhood of some $T_{i}$, repeated use of the techniques of the proof of Theorem 1 proves Theorem 3.

\section{REFERENCES}

1. J. W. Alexander, An example of a simply connected surface bounding a region which is not simply connected, Proc. Nat. Acad. Sci. U.S.A. 10 (1924), 8-10.

2. R. H. Bing, Approximating surfaces from the side, Ann. of Math. (2) 77 (1963), 145-192.

3. - Approximating surfaces with polyhedral ones, Ann. of Math. (2) 65 (1957), 456-483.

4. -—, A surface is tame if its complement is 1-ULC, Trans. Amer. Math. Soc. 101 (1961), 294-305.

5. - A wild surface each of whose arcs is tame, Duke Math. J. 28 (1961), 1-16.

6. - Conditions under which a surface in $E^{3}$ is tame, Fund. Math. 47 (1959), 105-139.

7. —_, Each disk in $E^{3}$ is pierced by a tame arc, Abstract 559-19, Notices Amer. Math. Soc. 6 (1959), 510.

8. - Locally tame sets are tame, Ann. of Math. (2) 59 (1954), 145-158.

9. —_ Notes of the topology institute, Univ. of Georgia, Athens, Ga., 1961. 
10. R. H. Fox and E. Artin, Some wild cells and spheres in three dimensional space, Ann. of Math. (2) 49 (1948), 979-990.

11. Joseph Martin, Disks on spheres, Abstract 584-9, Notices Amer. Math. Soc. 8 (1961), 498; Extending a disk on a sphere, Trans. Amer. Math. Soc. 109 (1963), 385-399.

12. E. E. Moise, Affine structures in 3-manifolds. VIII, Ann. of Math. (2) 59 (1954), 159-170.

13. L. S. Pontryjagin, Foundations of combinatorial topology, Graylock Press, Rochester, N. Y., 1952.

14. A. Schoenflies, Beitrage zur Theorie der Punktmengen. III, Math. Ann. 62 (1906), 286-328.

\author{
UNIVERSTTY OF MARYLAND, \\ College Park, Maryland \\ UNIVERSITY OF WISCONSIN, \\ Madison, Wisconsin
}

